\title{
Geographic and demographic variabilities of quantitative parameters in stress myocardial com- puted tomography perfusion
}

Jinoh Park ${ }^{1}$, Hyun-Sook Kim² ${ }^{2}$ Hye Jeon Hwang 3 , Dong Hyun Yang ${ }^{4}$, Hyun Jung Koo ${ }^{4}$, Joon-Won Kang ${ }^{4}$, and Young-Hak Kim ${ }^{1}$

\author{
${ }^{1}$ Division of Cardiology, Department \\ of Internal Medicine, Asan Medical \\ Center, University of Ulsan College \\ of Medicine, Seoul; ${ }^{2}$ Division of \\ Cardiology, Department of Internal \\ Medicine, ${ }^{3}$ Department of Radiology, \\ Hallym University Sacred Heart \\ Hospital, Anyang; ${ }^{4}$ Department of \\ Radiology, Asan Medical Center, \\ University of Ulsan College of \\ Medicine, Seoul, Korea
}

Received: January 4, 2016 Revised : January 19, 2016 Accepted: January 22, 2016

\section{Correspondence to}

Hyun-Sook Kim, M.D.

Division of Cardiology, Department of Internal Medicine, Hallym University Sacred Heart Hospital, 22 Gwanpyeong-ro 17obeon-gil, Dongan-gu, Anyang 14068, Korea

Tel: $+82-31-380-3979$

Fax: +82-31-386-2269

E-mail: hearthsk@hotmail.com
Background/Aims: To evaluate the geographic and demographic variabilities of the quantitative parameters of computed tomography perfusion (CTP) of the left ventricular (LV) myocardium in patients with normal coronary artery on computed tomography angiography (CTA).

Methods: From a multicenter CTP registry of stress and static computed tomography, we retrospectively recruited 113 patients (mean age, 60 years; $57 \mathrm{men}$ ) without perfusion defect on visual assessment and minimal $<20 \%$ of diameter stenosis) or no coronary artery disease on CTA. Using semiautomatic analysis software, quantitative parameters of the LV myocardium, including the myocardial attenuation in stress and rest phases, transmural perfusion ratio (TPR), and myocardial perfusion reserve index (MPRI), were evaluated in 16 myocardial segments.

Results: In the lateral wall of the LV myocardium, all quantitative parameters except for MPRI were significantly higher compared with those in the other walls. The MPRI showed consistent values in all myocardial walls (anterior to lateral wall: range, $25 \%$ to $27 \%$; $p=0.401$ ). At the basal level of the myocardium, all quantitative parameters were significantly lower than those at the mid- and apical levels. Compared with men, women had significantly higher values of myocardial attenuation and TPR. Age, body mass index, and Framingham risk score were significantly associated with the difference in myocardial attenuation.

Conclusions: Geographic and demographic variabilities of quantitative parameters in stress myocardial CTP exist in healthy subjects without significant coronary artery disease. This information may be helpful when assessing myocardial perfusion defects in CTP.

Keywords: Myocardial CT perfusion; Quantitative parameter; Geographic variability; Demographical variability

\section{INTRODUCTION}

Evaluation of not only anatomic obstructive lesions of the coronary artery but also the functional significance of the lesions is important when determining the treatment strategy for ischemic heart disease $[1,2]$. Revascu- larization of reversible myocardial ischemia based on thallium single-photon emission computed tomography (SPECT) has been used to improve the clinical outcomes of patients [1], although medical therapy has shown a treatment efficacy comparable to that of revascularization in small myocardial ischemia lesions 
involving less than $10 \%$ of the left ventricular (LV) myocardium [3]. Therefore, the use of functional imaging to assess myocardial ischemia is an emerging topic. Stress myocardial computed tomography perfusion (CTP) is one of the diagnostic tools used to detect myocardial ischemia, and is attracting attention because of its accuracy, convenience, and non-invasiveness [4].

Myocardial contrast enhancement can be evaluated by visual and quantitative analysis. Although visual analysis is the principal form of analysis used, an objective evaluation of CTP can be conducted by quantitative assessment of the ischemic burden of the myocardium [5]. Multiplanar reconstructions and minimum intensity projections of the LV myocardium have been used to compare myocardial perfusion defects with the level of perfusion of the normal myocardium. The transmural perfusion ratio (TPR) is derived from a comparison of the endomyocardial attenuation to that of the subepimyocardium [6]. The TPR shows good correlation with SPECT and invasive coronary angiography [7]. Both visual and quantitative analyses are based on an assessment of the attenuation of the contrast-enhanced LV myocardium; however, these methods can be disturbed by certain variables, including anatomical attenuation, gender-specific differences, different computed tomography (CT) scanner-derived factors, and CT artifacts [811]. One study has shown that the LV perfusion in the rest phase measured in asymptomatic patients was significantly higher in women than in men [10]. In 64-detector row CT, the normal myocardial perfusion in the LV myocardial septum showed higher attenuation than in the lateral and inferior walls [9]. Thus, in the present study, we aimed to identify the different attenuations of the LV myocardium according to demographic differences, and the myocardial segment, using a second-generation dual-source CT scanner in patients with normal or minimal coronary artery disease on computed tomography angiography (CTA).

\section{METHODS}

\section{Patients}

From a multicenter static CTP registry of dual-source $\mathrm{CT}$, which is a prospective registry of all patients who underwent CTP for the evaluation of chest pain, we ret- rospectively recruited 113 patients with normal CTP on visual assessment and minimal (<20\% diameter stenosis) or no coronary artery disease on CTA.

\section{CT imaging}

Stress-rest myocardial CTP protocols were performed using a second-generation dual-source CT scanner (Definition Flash, Siemens, Forchheim, Germany). Adenosine (Denosine, BCWP, Seoul, Korea) infusion (140 mg/kg/min for 5 minutes) was performed with retrospective electrocardiography-gated acquisition (Fig. 1). A bolus of iodinated contrast material (60 to $70 \mathrm{~mL}$, iomeprol; Iomeron 400, Bracco, Milan, Italy) was injected at a rate of $4.0 \mathrm{~mL} / \mathrm{sec}$. Then, $40 \mathrm{~mL}$ of a saline chaser was given. Ten minutes after the infusion of adenosine was discontinued, isosorbide dinitrate (Isoket Spray, UCB Pharma, Monheim, Germany) was sprayed into the oral cavity of the patient. Rest myocardial CTP was performed using the same protocol as that used for stress myocardial CTP. To reduce the radiation dose, the dose-pulsing window was reduced to $60 \%$ to $75 \%$ if the heart rate decreased to less than 70 beats/min after the adenosine infusion was stopped. The tube current-time product was modified in accordance with the body size of the patient. A smooth reconstruction kernel (B1of) was applied for perfusion imaging in both stress and rest CT data.

\section{Quantitative analysis of CTP}

LV myocardial attenuation was measured and analyzed using customized software (A-view Cardiac, Asan Medi-

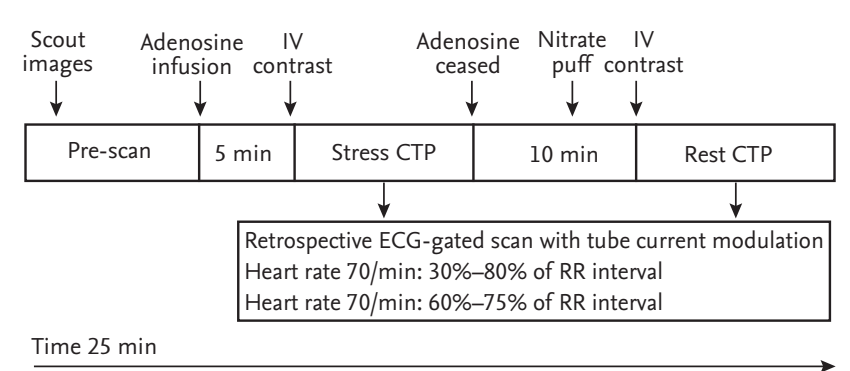

Figure 1. Protocol for computed tomography perfusion (CTP). After 5 minutes of intravenous (IV) adenosine infusion, the stress phase of myocardial CTP was initiated with IV infusion of contrast material. Ten minutes after stress myocardial CTP, the rest phase of myocardial CTP was initiated after administration of an oral spray of nitrate puff. The total time for the myocardial CTP imaging protocol was approximately 25 minutes. ECG, electrocardiography. 
cal Center, Seoul, Korea). The mid-diastolic phase of the CT data was used for the analysis. After semiautomatic segmentation of the LV myocardium, the mean attenuations of the 16 myocardial segments were measured at both stress (Density stress $_{\text {) }}$ ) and rest (Density ${ }_{\text {rest }}$ ) phases (Fig. 2). TPR was calculated for each segment as the ratio of the subendocardial attenuation density to the subepicardial attenuation on short-axis images of the LV myocardium [5]. Myocardial perfusion reserve index (MPRI) was calculated as the percentage difference in attenuation between stress and rest myocardial CTP images in each segment, as follows: (Density stress - Density $\left._{\text {rest }}\right) /$ Density ${ }_{\text {rest }} \times 100 \%$.

\section{Statistical analysis}

Continuous variables are presented as the mean \pm stan-
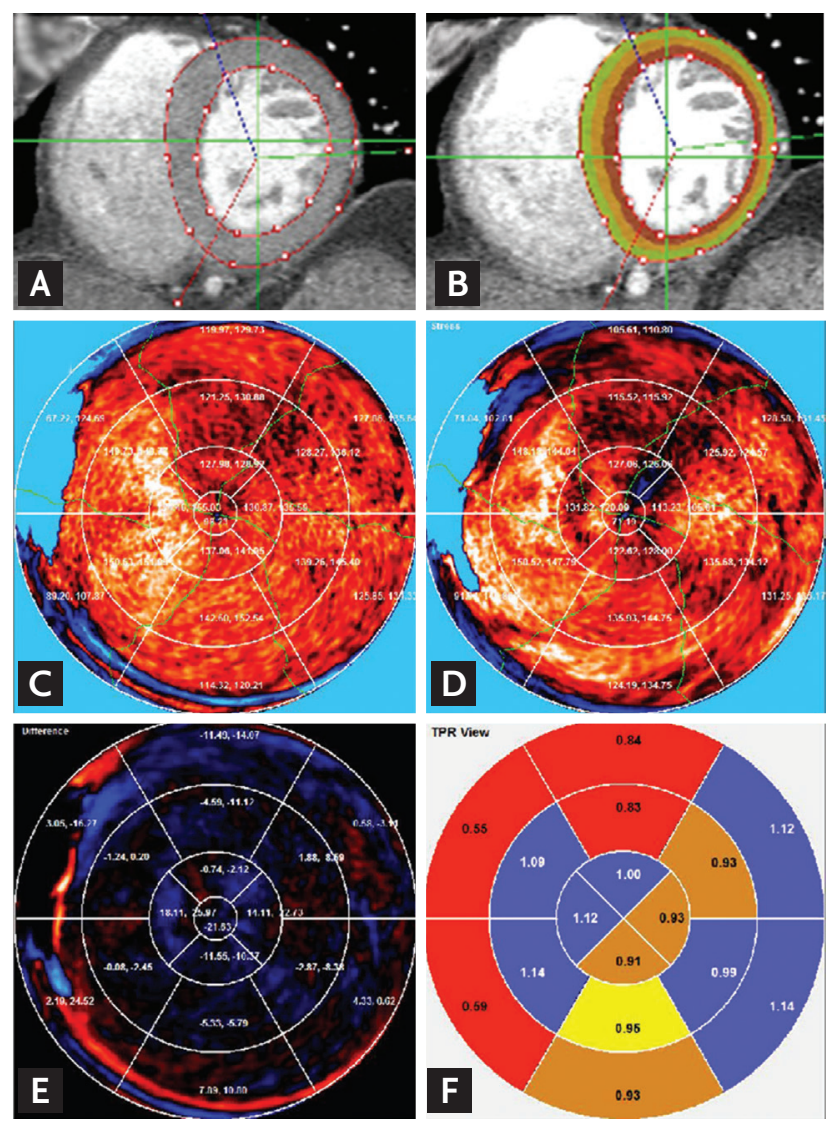

Figure 2. Short-axis slice of the left ventricular (LV) myocardium showing the semiautomatic methods for segmental and geographic myocardial evaluation. (A, B) The borders of the LV myocardium were drawn to demonstrate the endomyocardium to epimyocardium region. (C, D, E, F) The values of myocardial attenuation and transmural perfusion ratio (TPR) were automatically obtained. dard deviation. Using Student $t$ test, the myocardial attenuations in all segments were compared according to the anterior, septal, inferior, and lateral; apical, mid, and basal; and left anterior descending artery, right coronary artery, and left circumflex artery territories. Pearson correlation was used to identify any clinical factors related to the differences in myocardial attenuation. To evaluate gender differences in myocardial attenuation, Student $t$ test was used. Univariate and multivariable regression analyses were used to evaluate the relationship between the myocardial attenuation and clinical variables such as age, body mass index (BMI), and Framingham heart score (FHS). A p value less than 0.05 was regarded as statistically significant. All statistical analyses were performed using commercially available software SPSS version 22.0 (IBM Co., Armonk, NY, USA).

\section{RESULTS}

\section{Patient characteristics}

A total of 113 patients (aged $60 \pm 9.5$ years, 57 men) with normal CTP on visual assessment and minimal or no coronary artery disease were included in the present quantitative analysis. Patient characteristics are described in Table 1. Among them, 56 patients had hypertension and 20 patients had diabetes. No patient had pulmonary disease or LV dysfunction.

\section{Geometric variations in quantitative parameters}

The mean attenuations of the 16 myocardial segments in both rest and stress phases were displayed on a bull's eye map (Fig. 3). The mean attenuation of the myocardium was higher in the stress phase than in the rest phase (Fig. 4). All quantitative parameters except MPRI were significantly higher in the lateral wall than in the anterior, inferior, and septal walls (Table 2). The myocardial attenuation in the rest phase $(104 \pm 18$ Hounsfield unit $[\mathrm{HU}])$ was significantly higher in the lateral wall than in the anterior $(95 \pm 17 \mathrm{HU})$, septal $(98 \pm 25 \mathrm{HU})$, and inferior $(98 \pm 18 \mathrm{HU})$ walls. Even in the stress phase, the attenuation was significantly higher in the lateral wall $(127 \pm 19 \mathrm{HU})$ than in the anterior $(120 \pm 19 \mathrm{HU})$, septal $(120 \pm 27 \mathrm{HU})$, and inferior $(121 \pm 18 \mathrm{HU})$ walls. The TPR of the lateral wall was also higher than that of the other walls. No significant differences were observed in 
the MPRI of each myocardial wall, and consistent MPRI values were observed among the myocardial walls (an-

Table 1. Patient characteristics $(n=113)$

\begin{tabular}{lc}
\hline Variable & Value \\
\hline Age, yr & $60 \pm 9.5$ \\
Male sex & $57(50.4)$ \\
\hline Body mass index, kg/m ${ }^{2}$ & $25.4 \pm 2.7$ \\
Body surface area, $\mathrm{m}^{2}$ & $1.72 \pm 0.22$ \\
\hline Hypertension & $56(49.6)$ \\
Diabetes mellitus & $20(17.7)$ \\
Dyslipidemia & $53(46.9)$ \\
Smoking & \\
\hline Never-smoker & $45(39.8)$ \\
\hline Ex-smoker & $22(19.5)$ \\
\hline Current-smoker & $12(10.6)$ \\
Total cholesterol, mg/dL & $175.8 \pm 36.5$ \\
Triglyceride, mg/dL & $134.7 \pm 67.2$ \\
\hline LDL-C, mg/dL & $111.8 \pm 31.8$ \\
HDL-C, mg/dL & $50.5 \pm 13.4$ \\
Framingham risk score & $14.8 \pm 11.3$ \\
\hline Mild & $19(16.8)$ \\
\hline Moderate & $22(19.5)$ \\
\hline Severe & $22(19.5)$ \\
\hline
\end{tabular}

Values are presented as mean \pm standard deviation or number (\%).

LDL, low density lipoprotein cholesterol; HDL, high density lipoprotein cholesterol.

Total myocardium

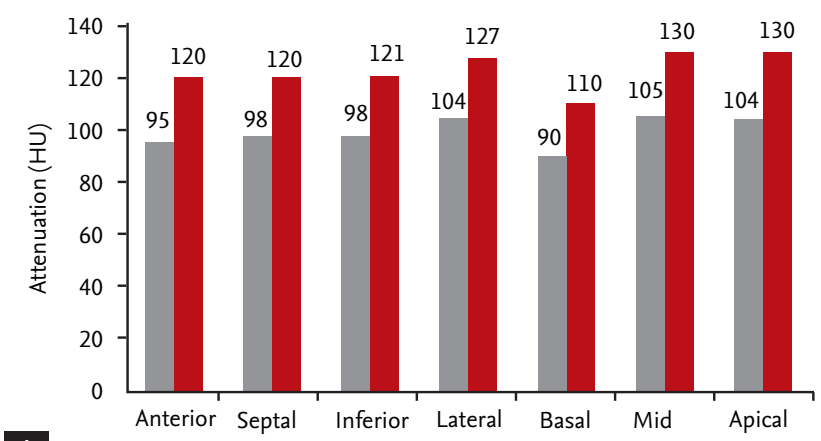

A

Rest phase Stress phase

Figure 4. Attenuation of the (A) total and (B) endomyocardial wall in the rest and stress phases. All $p$ values for the comparison of the measurements of the rest and stress phases are statistically significant $(p<0.001)$. HU, Hounsfield unit. terior to the lateral wall: range, $25 \%$ to $27 \% ; p=0.401$ ). These attenuation differences were also similarly noted in comparison with the coronary artery territories. The left circumflex arterial territory showed significantly different attenuation and TPR values than those shown by the left anterior descending artery and right coronary artery territories in both rest and stress phases.

Regarding the levels of LV myocardium, the myocardial attenuation at the basal level was significantly lower than that at mid-level and apical level in both rest and stress phases. The TPR was also significantly lower at the basal level.

\section{Demographic variations of quantitative parameters}

Among the binary clinical variables, gender had a significant effect on the values of all parameters except MPRI (Table 3). The myocardial density was higher in women

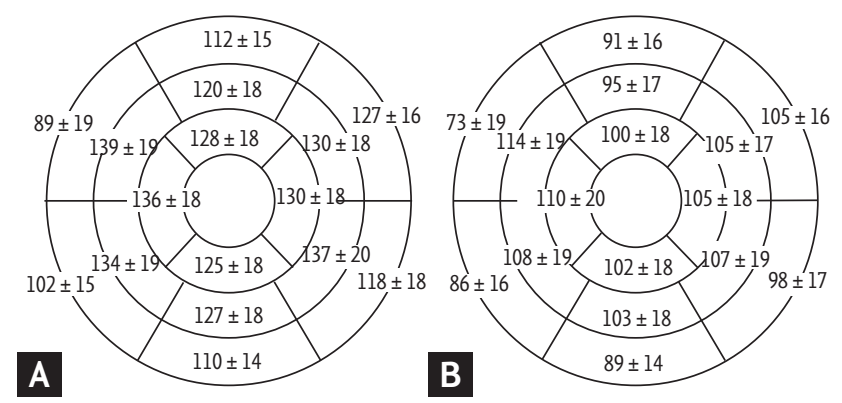

Figure 3. Normal myocardial attenuation in (A) the rest phase and (B) the stress phase in all patients.

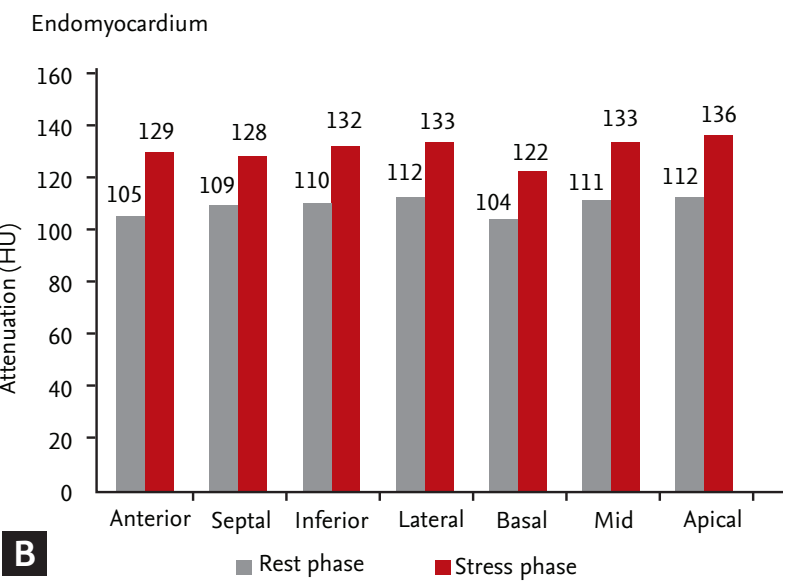


Table 2. Mean attenuation and quantitative parameters of the LV myocardium on myocardial computed tomography perfusion

\begin{tabular}{|c|c|c|c|c|c|c|c|c|}
\hline \multirow{2}{*}{ Variable } & \multicolumn{2}{|c|}{ Density $_{\text {rest }}, \mathrm{HU}$} & \multicolumn{2}{|c|}{ Density $_{\text {stress }}, \mathrm{HU}$} & \multicolumn{2}{|c|}{ TPR } & \multicolumn{2}{|c|}{ MPRI, \% } \\
\hline & Mean \pm SD & $p$ value & Mean \pm SD & $p$ value & Mean \pm SD & $p$ value & Mean \pm SD & $p$ value \\
\hline LV wall & & $<0.001$ & & $<0.001$ & & $<0.001$ & & 0.082 \\
\hline Anterior & $95 \pm 17$ & & $120 \pm 19$ & & $0.90 \pm 0.09$ & & $27 \pm 19$ & \\
\hline Septal & $98 \pm 25$ & & $120 \pm 27$ & & $0.97 \pm 0.19$ & & $24 \pm 18$ & \\
\hline Inferior & $98 \pm 18$ & & $121 \pm 18$ & & $0.89 \pm 0.09$ & & $25 \pm 18$ & \\
\hline Lateral & $104 \pm 18$ & & $127 \pm 19$ & & $1.02 \pm 0.09$ & & $26 \pm 19$ & \\
\hline LV level & & $<0.001$ & & $<0.001$ & & $<0.001$ & & $<0.001$ \\
\hline Basal & $90 \pm 19$ & & $110 \pm 20$ & & $0.90 \pm 0.17$ & & $22 \pm 18$ & \\
\hline Mid & $105 \pm 19$ & & $130 \pm 20$ & & $0.99 \pm 0.11$ & & $27 \pm 19$ & \\
\hline Apical & $104 \pm 19$ & & $130 \pm 19$ & & $0.99 \pm 0.10$ & & $28 \pm 19$ & \\
\hline Coronary territory & & $<0.001$ & & $<0.001$ & & $<0.001$ & & 0.198 \\
\hline LAD & $97 \pm 23$ & & $121 \pm 25$ & & $0.93 \pm 0.17$ & & $26 \pm 18$ & \\
\hline RCA & $98 \pm 19$ & & $120 \pm 21$ & & $0.92 \pm 0.12$ & & $24 \pm 18$ & \\
\hline LCX & $104 \pm 18$ & & $127 \pm 19$ & & $1.02 \pm 0.09$ & & $26 \pm 19$ & \\
\hline
\end{tabular}

LV, left ventricular; HU, Hounsfield unit; TPR, transmural perfusion ratio; MPRI, myocardial perfusion reserve index; LAD, left anterior descending artery; RCA, right coronary artery; LCX, left circumflex artery.

than in men in both rest and stress phases. Compared with men, women had significantly higher values of myocardial attenuation in both rest and stress phases, and also had higher TPR. There were no significant differences in all quantitative parameters irrespective of the presence of diabetes or hypertension, and smoking status. Multivariable regression analysis performed to evaluate the relationship between myocardial attenuation and linear clinical variables showed that age, BMI, and FHS were significantly associated with the difference in the myocardial attenuation (Table 4).

\section{DISCUSSION}

The findings of the present study indicate significant differences in the quantitative parameters of stress myocardial CTP in healthy subjects without coronary artery disease, according to geometric and demographic variables. The lateral wall of the LV myocardium showed a significantly higher attenuation and the basal level of the LV myocardium showed a lower attenuation than that shown by the other levels. The TPR was also higher in the lateral wall than in the other walls and was significantly lower at the basal level. However, there were no significant differences in the MPRI of the myocardial walls. In terms of gender-specific differences, myocardial attenuation was significantly higher in women than in men. By multivariable analysis, age, BMI, and FHS were significantly associated with myocardial attenuation. By considering the normal variabilities in the quantitative parameters of the CTP derived from the influence of these geographic and demographic parameters, confusion between true ischemia and normal variation can be prevented, and the diagnostic performance of CTP can be improved.

The results of our present study are in accordance with those of previous studies that reported variabilities in myocardial attenuation in CTP according to geometric and sex parameters [9,10,12]. However, the reasons for these variabilities are unclear. One possible explanation is that the BMI of women is lower than that of men. BMI is independently related to myocardial attenuation. Considering the differences in body size and blood volume between genders, a lower BMI and lower blood volume may cause faster circulation in the myocardium, resulting in high myocardial attenuation. We can presume that the reason for the differences in the myocardial perfusion in CTP between the sexes in our current analysis also explains the differences in the 
Table 3. Assessment of the relationship between the rest phase myocardial attenuation and clinical factors (binary variables)

\begin{tabular}{|c|c|c|c|c|c|c|c|c|}
\hline \multirow{2}{*}{ Variable } & \multicolumn{2}{|c|}{ Density $_{\text {rest }}, \mathrm{HU}$} & \multicolumn{2}{|c|}{ Density $_{\text {stress }}, \mathrm{HU}$} & \multicolumn{2}{|c|}{ TPR } & \multicolumn{2}{|c|}{ MPRI } \\
\hline & Mean \pm SD & $p$ value & Mean \pm SD & $p$ value & Mean \pm SD & $p$ value & Mean \pm SD & $p$ value \\
\hline Sex & & $<0.001$ & & $<0.001$ & & $<0.001$ & & 0.311 \\
\hline Male & $92.01 \pm 13.27$ & & $114.55 \pm 12.79$ & & $0.964 \pm 0.015$ & & $27.04 \pm 17.93$ & \\
\hline Female & $106.89 \pm 15.28$ & & $130.25 \pm 13.77$ & & $0.947 \pm 0.028$ & & $23.88 \pm 14.85$ & \\
\hline Diabetes & & 0.908 & & 0.793 & & 0.179 & & 0.795 \\
\hline Yes & $98.92 \pm 20.02$ & & $121.48 \pm 15.86$ & & $0.960 \pm 0.013$ & & $26.58 \pm 21.80$ & \\
\hline No & $99.48 \pm 15.23$ & & $122.51 \pm 15.38$ & & $0.955 \pm 0.026$ & & $25.23 \pm 15.24$ & \\
\hline Hypertension & & 0.083 & & 0.070 & & 0.594 & & 0.490 \\
\hline Yes & $95.74 \pm 16.45$ & & $119.68 \pm 14.98$ & & $0.96 \pm 0.018$ & & $25.56 \pm 18.34$ & \\
\hline No & $101.98 \pm 15.42$ & & $124.94 \pm 15.50$ & & $0.955 \pm 0.029$ & & $24.40 \pm 14.50$ & \\
\hline Smoking & & 0.756 & & 0.757 & & 0.475 & & 0.693 \\
\hline Yes & $100.83 \pm 18.15$ & & $121.16 \pm 11.54$ & & $0.959 \pm 0.023$ & & $22.86 \pm 15.96$ & \\
\hline No & $99.07 \pm 15.52$ & & $122.38 \pm 16.51$ & & $0.953 \pm 0.025$ & & $25.60 \pm 16.25$ & \\
\hline Dyslipidemia & & 0.216 & & 0.489 & & 0.712 & & 0.205 \\
\hline Yes & $97.26 \pm 16.74$ & & $121.18 \pm 15.53$ & & $0.957 \pm 0.021$ & & $27.65 \pm 20.24$ & \\
\hline No & $101.05 \pm 15.43$ & & $123.21 \pm 15.45$ & & $0.956 \pm 0.028$ & & $23.67 \pm 12.18$ & \\
\hline
\end{tabular}

HU, Hounsfield unit; TPR, transmural perfusion ratio; MPRI, myocardial perfusion reserve index.

pathophysiology and clinical presentation of ischemic heart disease between the sexes [13]. Similarly, estrogen affects the vascular tone in women [14]. In a previous report that adjusted for patient size and body surface area, no difference was observed in myocardial enhancement in CTP according to gender [9]. Decreased contrast e nhancement with an increase in BMI is a well-known phenomenon associated with obesity [15]. Thus, adjustment of the amount of contrast medium might be required for consistent enhancement of the myocardium, with consideration of the age, sex, and BMI of the patient. Regarding FHS, which showed a negative relationship with myocardial attenuation by multivariable analysis, we postulate that the presence of microvascular lesion that cannot be detected by CTA would affect the myocardial perfusion.

In a previous study, Crossett et al. [12] have reported that the lateral wall showed significantly lower attenuation in the normal LV myocardium in CTP. However, they measured LV attenuation by using a rectangular region of interest and did not follow the American Heart Association 17-segment model. Moreover, they did not measure the attenuation differences between the subendocardial, mid-myocardial, and subepicardial layers. As we included the entire LV myocardium and used dedicated software, the differences in our results from those of the previous study might be expected.

The present study had several limitations. First, we did not correlate the CTP results with those of other functional imaging modalities such as SPECT or stress echocardiography. Therefore, the perfusion pattern in individuals with a normal coronary artery may not be applicable to other perfusion imaging results. Second, our results might not be applicable to those of other CT scanners with low temporal resolution or other CTP protocols such as the rest-first protocol. However, our findings of normal differences in the geometric and demographic variables will help in the interpretation of CTP results. Third, we did not consider whether $\beta$-blocker use would affect coronary artery blood flow and, thus, myocardial perfusion. Fourth, our study is a retrospective analysis, which may have resulted in a selection bias. Older individuals can be expected to have a greater degree of diastolic dysfunction, which could result in microvascular dysfunction and less hyperemia on adenosine, manifesting as a lower myocardial attenuation on CTP.

Despite these limitations, we included a large study 
Table 4. Univariate and multivariable regression analysis to assess the relationship between the rest phase and the stress phase myocardial attenuation and clinical factors (linear variables)

\begin{tabular}{|c|c|c|c|c|c|c|}
\hline \multirow{2}{*}{ Variable } & \multicolumn{3}{|c|}{ Univariate } & \multicolumn{3}{|c|}{ Multivariable } \\
\hline & $R^{2}$ & $95 \% \mathrm{CI}$ & $p$ value & $\mathrm{B}$ & $95 \% \mathrm{CI}$ & $p$ value \\
\hline \multicolumn{7}{|l|}{ Rest phase } \\
\hline Age, yr & 0.045 & 0.05 to 0.67 & 0.023 & 0.612 & 0.19 to 1.03 & 0.005 \\
\hline $\mathrm{BMI}, \mathrm{kg} / \mathrm{m}^{2}$ & 0.209 & -3.61 to -1.59 & $<0.001$ & -2.159 & -3.56 to -0.76 & 0.003 \\
\hline TG, mg/dL & 0.030 & -0.09 to 0.01 & 0.088 & & & \\
\hline HDL-C, mg/dL & 0.005 & -0.15 to 0.32 & 0.481 & & & \\
\hline LDL-C, mg/dL & 0.019 & -0.17 to 0.04 & 0.195 & & & \\
\hline FRS & 0.078 & -0.75 to -0.05 & 0.026 & -0.364 & -0.72 to -0.01 & 0.047 \\
\hline $\mathrm{HR}, / \min$ & 0.000 & -0.30 to 0.38 & 0.825 & & & \\
\hline $\mathrm{SBP}, \mathrm{mmHg}$ & 0.009 & -0.08 to 0.25 & 0.328 & & & \\
\hline $\mathrm{DBP}, \mathrm{mmHg}$ & 0.034 & -0.58 to 0.00 & $0.05^{2}$ & & & \\
\hline \multicolumn{7}{|l|}{ Stress phase } \\
\hline Age, yr & 0.073 & 0.15 to 0.74 & 0.004 & 0.667 & 0.31 to 1.03 & $<0.001$ \\
\hline $\mathrm{BMI}, \mathrm{kg} / \mathrm{m}^{2}$ & 0.327 & -4.16 to -2.31 & $<0.001$ & -2.873 & -4.08 to -1.67 & $<0.001$ \\
\hline TG, mg/dL & 0.025 & -0.08 to 0.01 & 0.119 & & & \\
\hline HDL-C, mg/dL & 0.045 & 0.02 to 0.47 & 0.036 & & & \\
\hline LDL-C, mg/dL & 0.000 & -0.10 to 0.10 & 0.985 & & & \\
\hline FRS & 0.090 & -0.75 to -0.08 & 0.017 & -0.339 & -0.65 to -0.03 & 0.032 \\
\hline $\mathrm{HR}, / \mathrm{min}$ & 0.000 & -0.24 to 0.22 & 0.917 & & & \\
\hline $\mathrm{SBP}, \mathrm{mmHg}$ & 0.002 & -0.18 to 0.11 & 0.630 & & & \\
\hline DBP, $\mathrm{mmHg}$ & 0.015 & -0.48 to 0.10 & 0.198 & & & \\
\hline
\end{tabular}

The $r^{2}$ was 0.286 for the three significant parameters obtained by multivariable regression analysis.

CI, confidence interval; BMI, body mass index; TG, triglyceride; HDL-C, high density lipoprotein cholesterol; LDL-C, low density lipoprotein cholesterol; FRS, Framingham risk score; HR, heart rate; SBP, systolic blood pressure; DBP, diastolic blood pressure.

population and evaluated the variability of myocardial attenuation on CTP. Moreover, via a comparison of stress and rest myocardial attenuation, TPR and MPRI values in the normal CTP were thoroughly evaluated. Notably, the TPR also showed geometric and demographic variability. However, the MPRI did not show any significant variability. As the MPRI has been shown to be useful in other modalities such as cardiac magnetic resonance [16], further studies of its usefulness in CTP would be worthwhile. If the MPRI is shown to be effective in the evaluation of myocardial perfusion, its features could conceivably be used in the assessment of CTP without the need to consider geographic and demographic factors.

In conclusion, the lateral wall and basal level of the LV myocardium shows significantly different quantitative parameters from those of the other regions. Myocardial attenuation can vary with the gender, age, BMI, or FHS of the patient. This information may be useful for the assessment of myocardial perfusion defects in CTP.

\section{KEY MESSAGE}

1. Stress myocardial computed tomography perfusion (CTP) in patients without coronary artery disease shows higher quantitative parameter values (myocardial attenuation, transmural perfusion ratio [TPR]) in the lateral wall of the left ventricular (LV) myocardium and lower quantitative parameter values (myocardial attenuation, TPR, myocardial perfusion reserve 
index) at the basal level of the LV myocardium.

2. Stress myocardial CTP in patients without coronary artery disease shows higher attenuation of the LV myocardium in women than in men.

3. There is a significant correlation between the attenuation of the LV myocardium and certain clinical variables, namely, age, body mass index, and Framingham cardiovascular risk score.

4. Consideration of normal variabilities in the quantitative parameters of stress myocardial CTP caused by these geographic and demographical parameters will be useful in the assessment of CTP.

\section{Conflict of interest}

No potential conflict of interest relevant to this article was reported.

\section{REFERENCES}

1. Kim YH, Ahn JM, Park DW, et al. Impact of ischemia-guided revascularization with myocardial perfusion imaging for patients with multivessel coronary disease. J Am Coll Cardiol 2012;60:181-19o.

2. Fearon WF. Physiologic approach for coronary intervention. Korean J Intern Med 2013;28:1-7.

3. Hachamovitch R, Hayes SW, Friedman JD, Cohen I, Berman DS. Comparison of the short-term survival benefit associated with revascularization compared with medical therapy in patients with no prior coronary artery disease undergoing stress myocardial perfusion single photon emission computed tomography. Circulation 2003;107:2900-2907.

4. Ko BS, Cameron JD, Meredith IT, et al. Computed tomography stress myocardial perfusion imaging in patients considered for revascularization: a comparison with fractional flow reserve. Eur Heart J 2012;33:67-77.

5. Ko BS, Cameron JD, Leung M, et al. Combined CT coronary angiography and stress myocardial perfusion imaging for hemodynamically significant stenoses in patients with suspected coronary artery disease: a comparison with fractional flow reserve. JACC Cardiovasc Imaging 2012;5:1097-1111.

6. George RT, Arbab-Zadeh A, Miller JM, et al. Adenosine stress 64- and 256-row detector computed tomography angiography and perfusion imaging: a pilot study evaluating the transmural extent of perfusion abnormalities to predict atherosclerosis causing myocardial ischemia. Circ Cardiovasc Imaging 2009;2:174-182.

7. Cury RC, Magalhaes TA, Paladino AT, et al. Dipyridamole stress and rest transmural myocardial perfusion ratio evaluation by 64 detector-row computed tomography. J Cardiovasc Comput Tomogr 2011;5:443-448.

8. Kitagawa K, George RT, Arbab-Zadeh A, Lima JA, Lardo AC. Characterization and correction of beam-hardening artifacts during dynamic volume CT assessment of myocardial perfusion. Radiology 2010;256:111-118.

9. Stanton CL, Haramati LB, Berko NS, et al. Normal myocardial perfusion on 64 -detector resting cardiac CT. J Cardiovasc Comput Tomogr 2011;5:52-6o.

10. Byrne C, Kuhl JT, Zacho M, et al. Sex- and age-related differences of myocardial perfusion at rest assessed with multidetector computed tomography. J Cardiovasc Comput Tomogr 2013;7:94-101.

11. Rodriguez-Granillo GA, Rosales MA, Degrossi E, Rodriguez AE. Signal density of left ventricular myocardial segments and impact of beam hardening artifact: implications for myocardial perfusion assessment by multidetector CT coronary angiography. Int J Cardiovasc Imaging 2010;26:345-354.

12. Crossett MP, Schneider-Kolsky M, Troupis J. Normal perfusion of the left ventricular myocardium using 320 MDCT. J Cardiovasc Comput Tomogr 2011;5:406-411.

13. Vaccarino V, Badimon L, Corti R, et al. Ischaemic heart disease in women: are there sex differences in pathophysiology and risk factors? Position paper from the working group on coronary pathophysiology and microcirculation of the European Society of Cardiology. Cardiovasc Res 2011;90:9-17.

14. Mendelsohn ME, Karas RH. The protective effects of estrogen on the cardiovascular system. N Engl J Med 1999;340:1801-1811.

15. Bae KT, Seeck BA, Hildebolt CF, et al. Contrast enhancement in cardiovascular MDCT: effect of body weight, height, body surface area, body mass index, and obesity. AJR Am J Roentgenol 2008;190:777-784.

16. Cullen JH, Horsfield MA, Reek CR, Cherryman GR, Barnett DB, Samani NJ. A myocardial perfusion reserve index in humans using first-pass contrast-enhanced magnetic resonance imaging. J Am Coll Cardiol 1999;33:1386-1394. 